\title{
Collagen Corneal Cross-linking in a Keratoconic Eye with Diffuse Corneal Edema
}

\author{
Ramez Barbara, Hanna Garzozi, Adel Barbara, Yossef Pickel, Ankur Barua
}

\begin{abstract}
Corneal cross-linking has proven safety and efficacy in arresting the progression of keratoconus. Its use has been extended to resolution of corneal edema. We present a case report and review of literature on the treatment of corneal edema using corneal cross-linking. Corneal cross-linking seems to be an effective method in reducing corneal edema at least as a temporary measure till definitive solution, such as penetrating keratoplasty (PKP) or Descemet's stripping automated endothelial keratoplasty (DASEK) is preformed.
\end{abstract}

Keywords: Cornea, Corneal edema, Collagen Corneal crosslinking, Collagen, Topography, Corneal dystrophy, Orbscan, Keratoconus, Corneal thickness, Glaucoma, Intraocular Pressure.

How to cite this article: Barbara R, Garzozi H, Barbara A, Pickel $Y$, Barua A. Collagen Corneal Cross-linking in a Keratoconic Eye with Diffuse Corneal Edema. Int J Kerat Ect Cor Dis 2012;1(2):134-139.

\section{Source of support Nil}

Conflict of interest: None

\section{INTRODUCTION}

Collagen corneal cross-linking (CXL) is gaining popularity as a treatment for arresting the progression of keratoconus since the first clinical result publication in 2003 by Theo Seiler's group. CXL increases the corneal biomechanical strength by increasing the interfibrillar covalent bonds. ${ }^{1}$ It increases collagen fibrils thickness, decreases the potential space for water accumulation and decreases sw elling rate. ${ }^{2}$

Wollensak et al reported on the hydration behavior of the cross-linked cornea. He demonstrated increased resistance of the anterior cross-linked cornea to the diffusion of water from the posterior to the anterior cornea. ${ }^{3}$

Recently, there have been various reports on the use of $\mathrm{CXL}$ as a treatment for bullous keratopathy (BK) as a result of Fuch's dystrophy, glaucoma, pseudophakic BK and following penetrating keratoplasty. CXL reduces corneal edema, corneal thickness and epithelial bullae consequently improving symptoms, such as pain and improving visual acuity (VA) and photophobia. ${ }^{4-11}$

\section{LITERATURE REVIEW}

There are various ways of performing $C X L$ for $B K$.

1. The traditional way reported by Seiler's group; ${ }^{1}$ the epithelium, is removed from the central 8 to $9 \mathrm{~mm}$ of the cornea. Riboflavin $0.1 \%$ with dextran $20 \%$ is instilled until the appearance of yellow flare in the anterior chamber (AC). The cornea is irradiated with U Itraviol et A (UV A), (365-370 nm) light source $5 \mathrm{~cm}$ from the eye with a light intensity of $3 \mathrm{~mW} / \mathrm{cm}^{2}$ for 30 minutes. During treatment riboflavin and balanced salt solution (BSS) are instilled into the eye.

2. Glucose $40 \%$ is instilled to the eye 1 day prior to the procedure in order to reduce corneal thickness ${ }^{8}$ followed by the same procedure as in 1 .

3. Glycerin $70 \%$ in instilled to the eye till achievement of corneal thickness of 340 to $370 \mu$ prior to the start of the procedure as in $1 .^{12}$

4. Two pockets are created with femtosecond laser. The epithelium is not removed and riboflavin $0.2 \mathrm{ml}$ of $0.1 \%$ are injected in the pockets and then the cornea is exposed to UV A (365-370 nm) light source $5 \mathrm{~mm}$ from the eye with a light intensity of $15 \mathrm{~mW} / \mathrm{cm}^{2}$ for 7 minutes. ${ }^{9}$ The advantage of this method is that it painless as the epithelium is not removed.

Ehlers et al. ${ }^{4,5}$ reported on decreased corneal edema in 10 out of 11 eyes treated with CXL. VA improved in most eyes following $C X L$. In one eyes $V A$ improved from 0.04 to 0.4 on decimal notation after 6 months and this improvement was maintained at the 17 months follow-up (FU). G hanem et al ${ }^{6}$ reported on 14 eyes with pseudophakic bollous keratopathy (PBK) in which the mean corneal thickness decreased following CXL from 747 to $623 \mu$ at 1 month FU. Six months after treatment, mean corneal thickness increased to $710 \mu$ but remained less than the preoperative thickness. No changes in pain score were reported in this study.

K ozobolis et $\mathrm{al}^{7}$ reported on 2 eyes with PBK with FU of 2 months with decreased corneal edema and improvement in VA following CXL. W ollensak et al ${ }^{8}$ reported on 3 eyes with corneal edema as a result of $B K$ with a mean decrease in corneal thickness of $94 \pm 14 \mu$ after 18 months. In cases where the cornea was not cicatrized there was improvement in V A. K rueger et al ${ }^{9}$ reported on 1 eye with corneal edema with a preoperative corneal thickness of $675 \mu$ and VA of counting fingers (CF). Following $C X L$ treatment corneal thickness was reduced to $550 \mu$ and VA improved to $6 / 24$, 6 months following treatment.

B arbosa et al ${ }^{10}$ reported on reduction in corneal edema and improvement in symptoms in 11 of 25 treated eyes $(44 \%)$ after 6 months. The reduction in corneal edema was significant at 3 months following treatment. In $56 \%$ of eyes 
Collagen Corneal Cross-linking in a Keratoconic Eye with Diffuse Corneal Edema

there was recurrence of bullae after 6 months. Gadelha et al ${ }^{11}$ reported on two eyes with PBK in which pain was reduced following treatment. However, there was no significant reduction in corneal thickness or improvement in VA. Hafezi et $\mathrm{al}^{12}$ reported on $C X L$ treatment for fluctuations in $V A$ as a result of Fuch's endothelial dystrophy (FED). The authors reportimprovement in V A and V A fluctuations, and decrease in corneal edema in a $\mathrm{FU}$ of 3 months.

\section{CASE REPORT}

A 28-year-old male was presented to our medical center in February 2010. After reviewing his medical records we revealed that he was first presented to his ophthalmologist in October 2008 with uncorrected visual acuity (UCV A) of $6 / 6$ in his right eye and best spectacle-corrected visual acuity (BSCVA) of $6 / 7$ with refractive correction of -1.25 to $0.50 \times 165$. In December 2009, he had a further visit to his ophthalmologist. He was noted to have UCV A of $6 / 6$ in his right eye and BSCVA $6 / 9$ with refractive correction of 0.75 to $2.00 \times 165$. Slit-lamp examination was unremarkable at that stage but he was suspected to have keratoconus. Two weeks later, he presented again with further deterioration in his subjective VA. On examination, his UCV A was 6/30 and BSCVA of $6 / 15$. On slit-lamp examination, he was suspected to have corneal edema. Endothelial cell count (ECC) was 3,106 in his right eye and 2,538 in his left eye with an edematous cornea and a normal appearing cell morphology. He was diagnosed with keratoconus and diffuse corneal edema (not hydrops). He was treated with PredForte 1\% (prednisolone acetate) and Cosopt (Dorzolamide Hydrochloride-Timolol $M$ aleate) and because of further decrease in VA and his unresponsiveness to medical treatment he was listed for PKP.

In February 2010, he presented to our medical center with UCVA of CF from 1.5 meters and BSCVA of $6 / 60-2$ with correction of $-6.00 \times 165$. Slit-lamp examination of his left eye revealed quite eye with normal appearing conjunctivae, diffuse corneal edema with no Descemet rupture, Descemet membrane opacities or keratic precipitates (KP). He had a normal anterior chamber (AC), clear lens and normal fundus examination including optic disk. H is right eye slit-lamp examination was unremarkable with a normal fundus. Central corneal thickness (CCT) was $658 \mu$ in his left eye and $572 \mu$ in his right eye. Corneal topography of the left eye was suggestive of keratoconus with a normal topography of the right eye (Figs 1 to 3). He had normal ocular response analyzer (ORA) signals in the right eye and abnormal in the left eye with an IOPcc of 11.1 and $20.6 \mathrm{~mm} \mathrm{H}$ g respectively (Fig. 4) X al acom (L atanoprost $0.05 \%+$ Timolol $0.5 \%$ ) and PredF orte $1 \%$ (prednisone) were

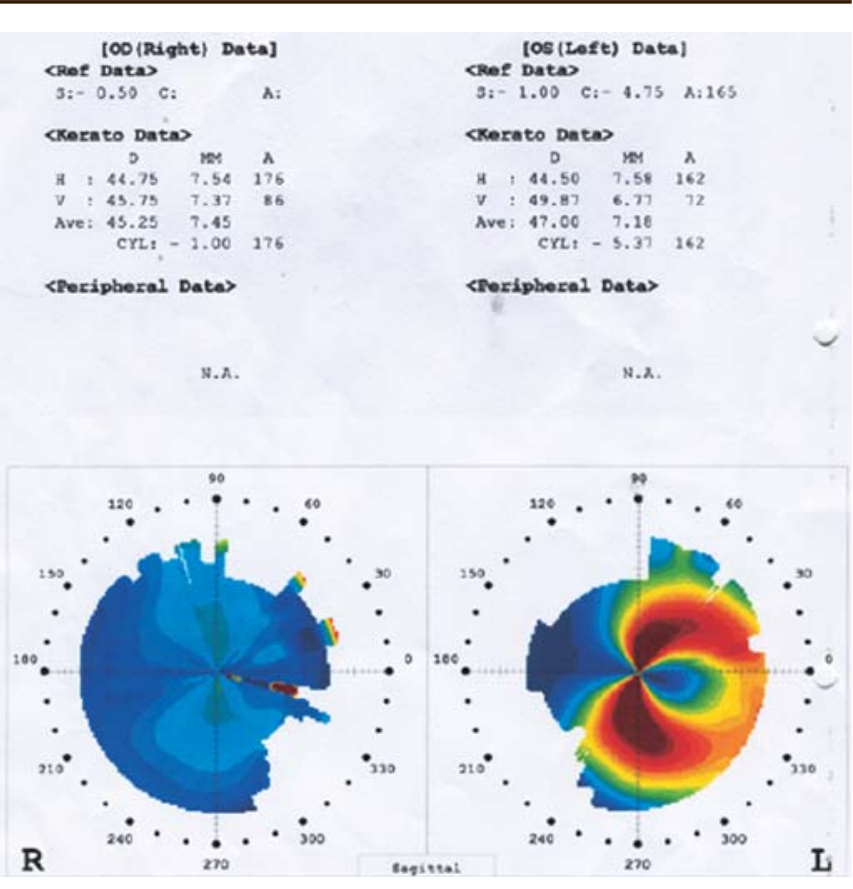

Fig. 1: Preoperative topography suggesting $\mathrm{KC}$ with high $\mathrm{K}$ readings of the left eye and a normal topography of the right eye

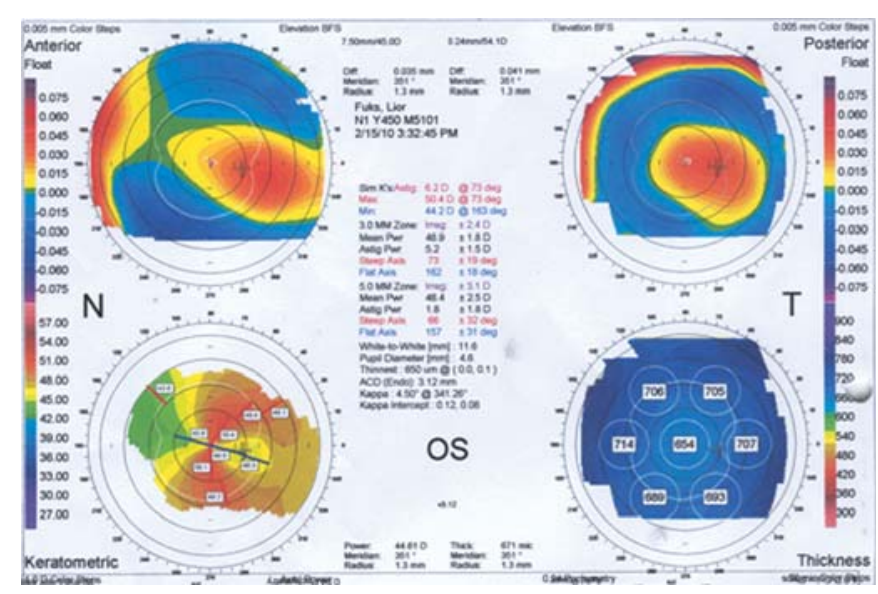

Fig. 2: Preoperative orbscan demonstrating thick cornea with high posterior float and anterior float of the left eye

prescribed till the next FU a week later. No improvement was noted in the subsequent FU a week later. We discussed the situation with the patient and made him aware that PK $P$ can al ways be done at any stage, but we suggested $C X L$.

A that stage he underwent $C X L$ to his left eye with of view of reducing his corneal edema. We used the surgical technique recommended by Theo Seiler and his group. Surgical procedure was performed by one surgeon under topical anesthesia. Standard prepping and draping were performed. The eye was washed with polidine $4 \%$. A $9 \mathrm{~mm}$ radius central corneal epithelium was removed. The patient was seated and riboflavin $0.1 \%$ combined with dextran $20 \%$ was instilled every 5 minutes for 30 minutes. The exposure of the cornea to UVA was done only after the appearance of a strong yellow flare in AC. Speculum was inserted and 


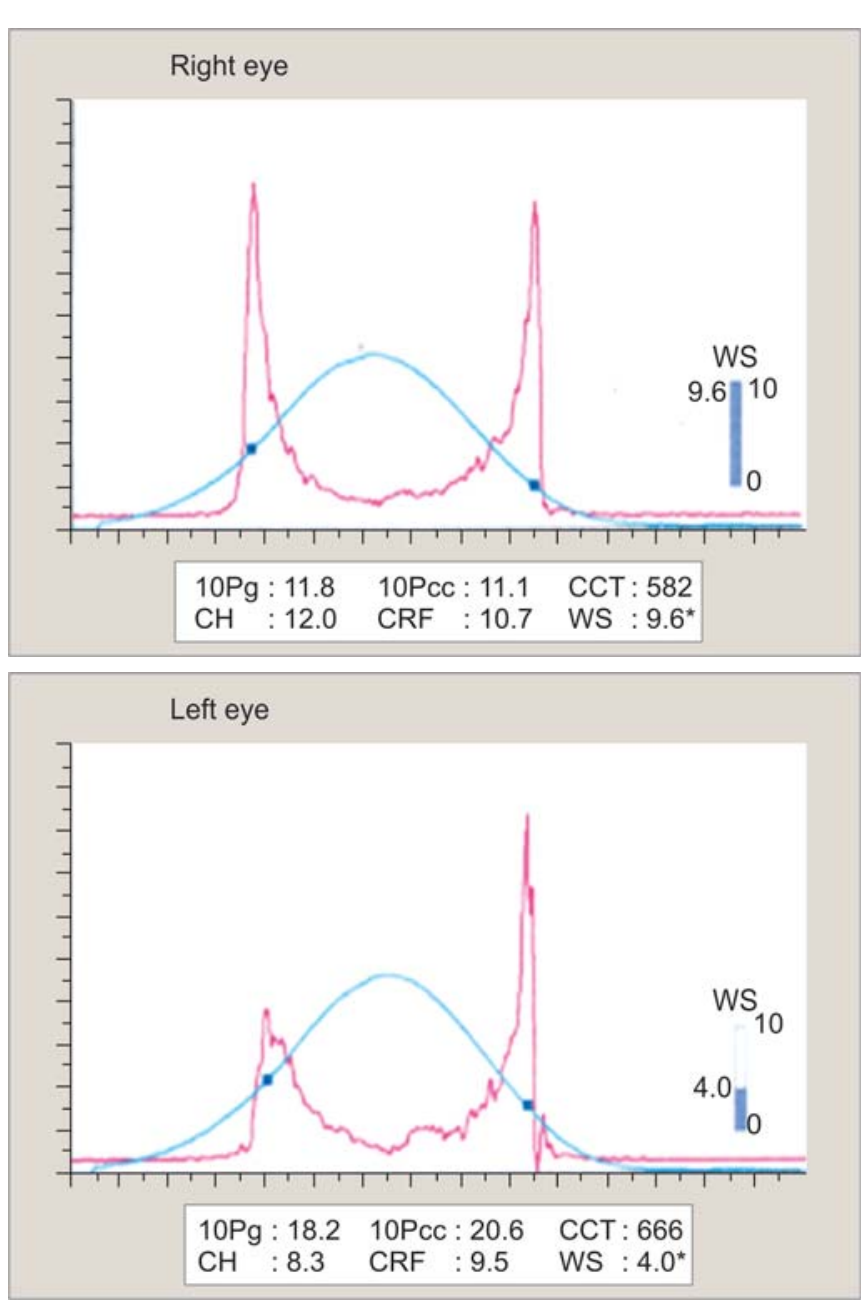

Fig. 3: Ocular response analyzer (ORA). Upper: Demonstrating regular pattern with corneal resistance factor (CRF) and corneal hysteresis $(\mathrm{CH})$ within normal limits in the right eye. Lower: Demonstrating irregular pattern with corneal resistance factor and corneal hysteresis lower than normal but as expected in keratoconic eye and edematous cornea

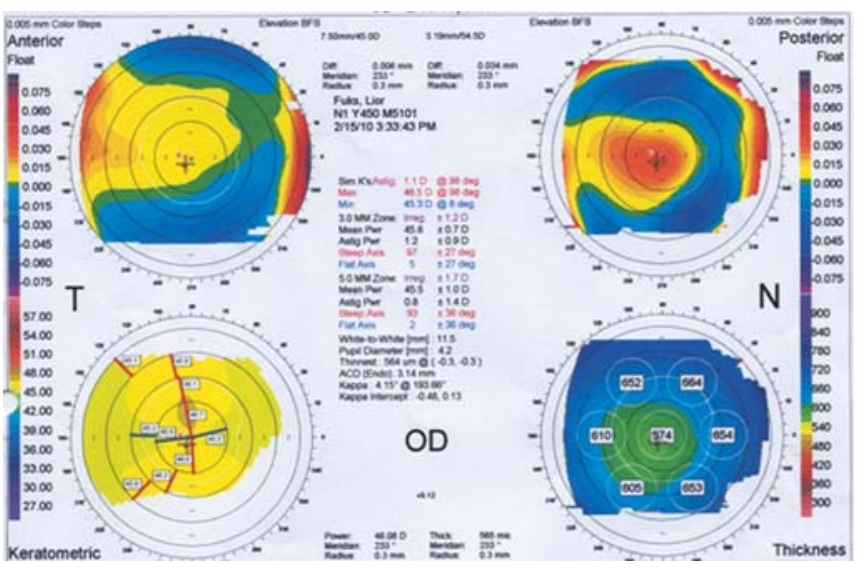

Fig. 4: Normal orbscan of the right eye

the patient was asked to look into UV A $(365-370 \mathrm{~nm})$ light source $5 \mathrm{~mm}$ from the eye (IROC UVX) with a light intensity of $3 \mathrm{~mW} / \mathrm{cm}^{2}$. During treatment riboflavin was instilled every 5 minutes into the eye and BSS every 3 minutes for further 30 minutes. Following the procedure contact lens
(CL) was applied until full epithelialization. Vigamox (moxifloxacin $0.5 \%$ ) antibiotics were prescribed until full epithelialization which occurred after 3 days. A PredForte 6 time daily for 2 weeks was used and was tapered gradually. $\mathrm{He}$ was asked to continue his Xalacom. Valtrex (valacyclovir) $1 \mathrm{gm} 3$ times daily for 1 week was prescribed.

Five days postoperatively (PO) his BSCV A was CF at 1 meter with a CCT of $682 \mu$. Corneal topography with computerized corneal topography KR 7000P (Topcon) was not recordable.

Three weeks PO his UCV A was $6 / 60$ and BSCV A of $6 /$ $18+$ with -1.00 to $2.50 \times 165$ correction. CCT of $572 \mu$ in the right eye and $590 \mu$ in the left eye with an IOPcc of 10.5 and $18.8 \mathrm{~mm} \mathrm{Hg}$ respectively. Five weeks PO his CCT was $577 \mu$ in the left eye and a bullae appeared in the inferiotemporal cornea with a CCT of $751 \mu$ over the bullae. Three months PO his UCVA was $6 / 48$ and BSCVA of $6 /$ 15 - with $-2.5 \times 160$ correction and CCT of $568 \mu$ in the left eye.

A t 4.5 months UCV A was $6 / 60$ and BSCV A was $6 / 12$ with +0.25 to $2.75 \times 160$ correction. He was noted to have haze +1 (Fig. 5). His corneal topography of $\mathrm{K}$ minimum of 43.37, $\mathrm{K}$ maximum of 47.12 and $\mathrm{K}$ average of 45.12 (Fig. 6). The CCT in the right eye was 568 and $559 \mu$ in the left eye with ECC of 3,247 and 2,591 respectively. CCT in the inferior temporal area over the former bullae was 688 with a scar over the area (Fig. 7) The IOPcc was $12.2 \mathrm{~mm} \mathrm{Hg}$ in the left eye (Fig. 8). He was prescribed FM L twice daily and continued $X$ alacom once a day.

The cornea and the UCVA, BSCVA, CCT, IOP and refraction were stable and the treatment (eyedrops) were discontinued.

Here is his last FU in a N ovember 2011: BSCVA 6/9 partial, refraction plano $=-2.5 \times 160$ and IOPCC $12.9 \mathrm{~K}$ minimum $43.62, \mathrm{~K}$ maximum $47.87, \mathrm{~K}$ average $45.62, \mathrm{CCT}$ left eye $590 \mu$, right eye $564 \mu$ (Figs 9 and 10).

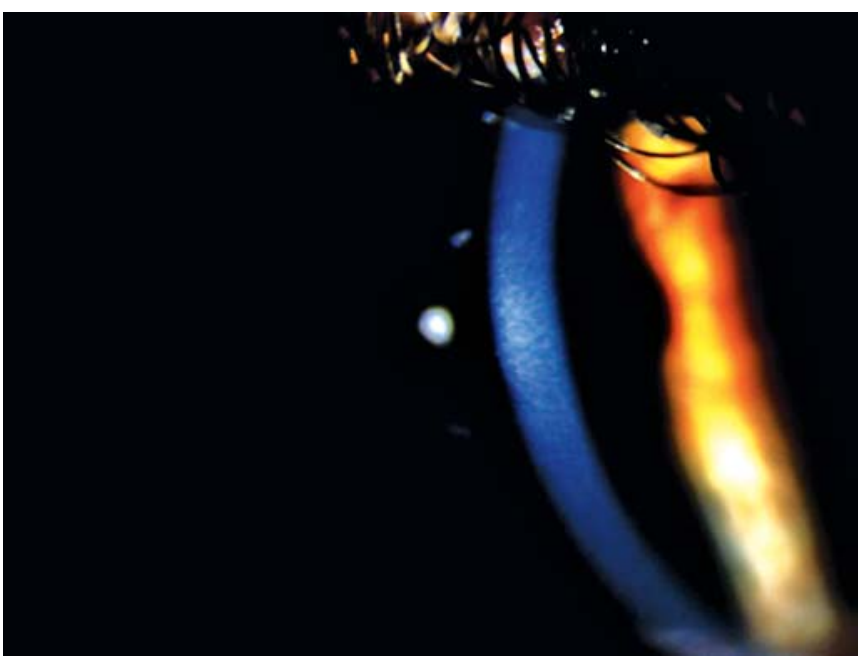

Fig. 5: Postoperative corneal haze 


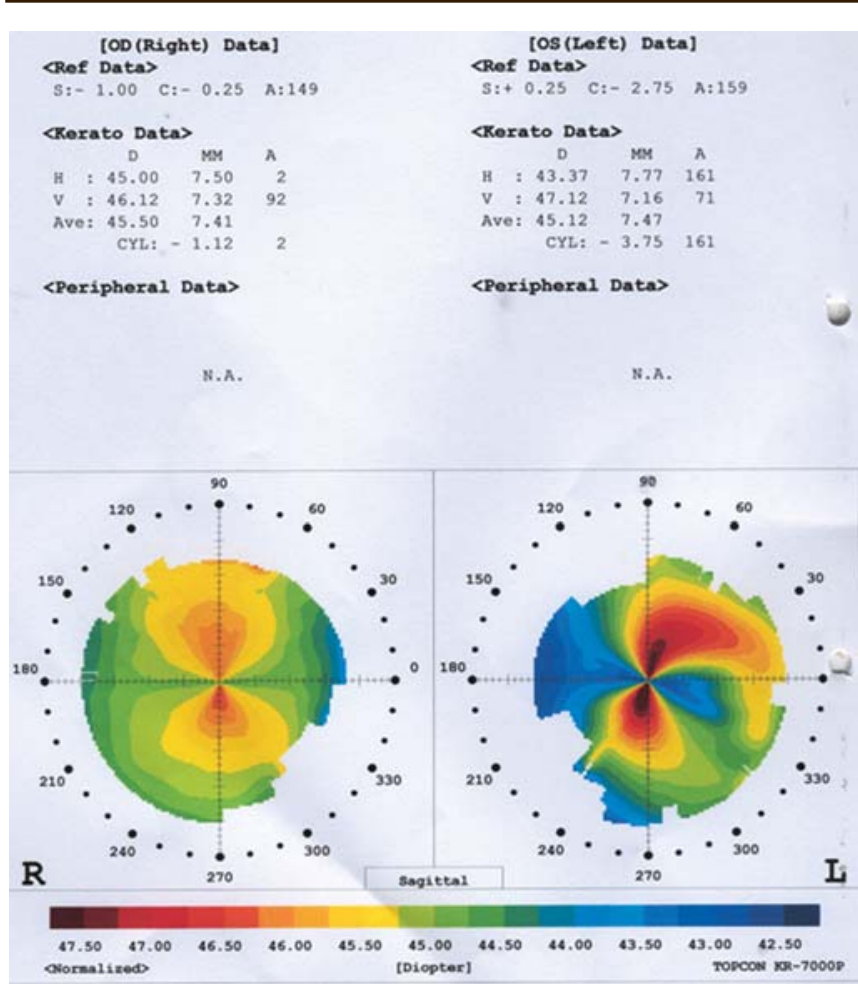

Fig. 6: Postoperative topography showing reduced $\mathrm{K}$ readings and improvement in corneal regularity of the left eye

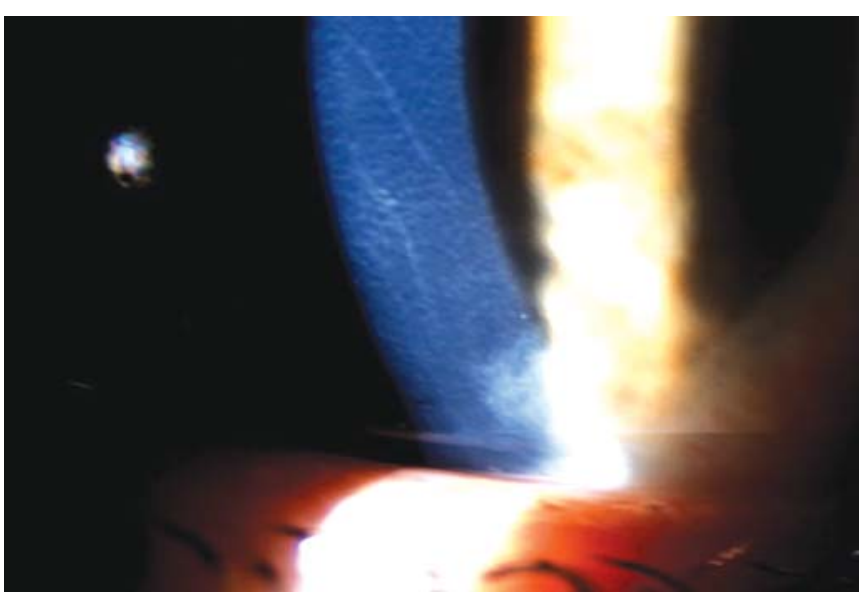

Fig. 7: Postoperative corneal haze with a scar over the previous bullae in the inferiotemporal cornea

\section{DISCUSSION}

A mong the methods of $C X L$ for treating corneal edema mentioned in the literature review. The method described by $\mathrm{H}$ afezi et al seems to us the most suitable for treating corneal edema as the treatment is supposed to cover the major part of the stroma and not only the anterior part as in the classical methods. As we know, CXL affects the first $300 \mu$ of the cornea and in an edematous cornea the treated stoma may constitute a negligible part of the anterior 'real anterior stoma' because of the huge edema. W e performed the treatment according to the classical method because we were not aware of the method describe by $\mathrm{H}$ afezi et al which was published in May 2010 and our treatment was
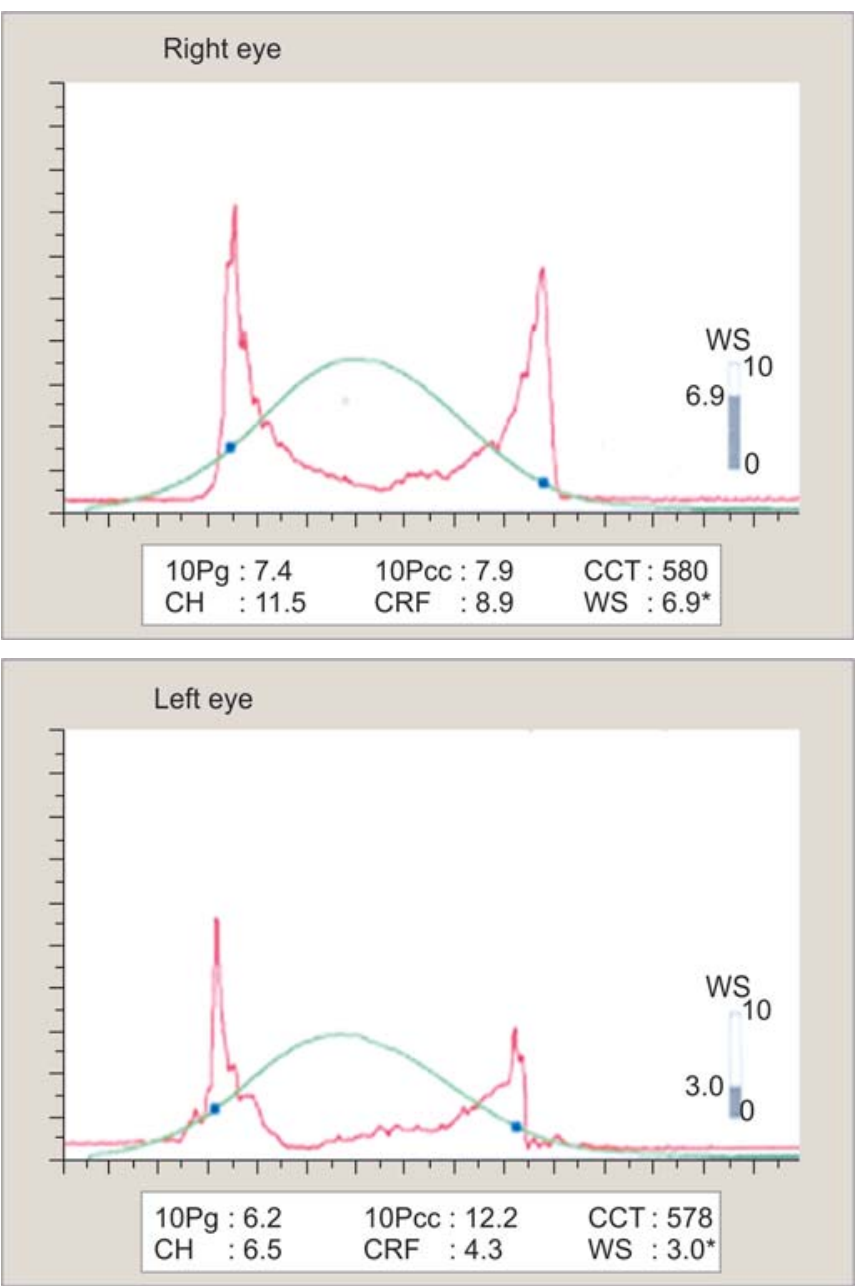

Fig. 8: Ocular response analyzer (ORA). Demonstrating regularization of the pattern in the left eye

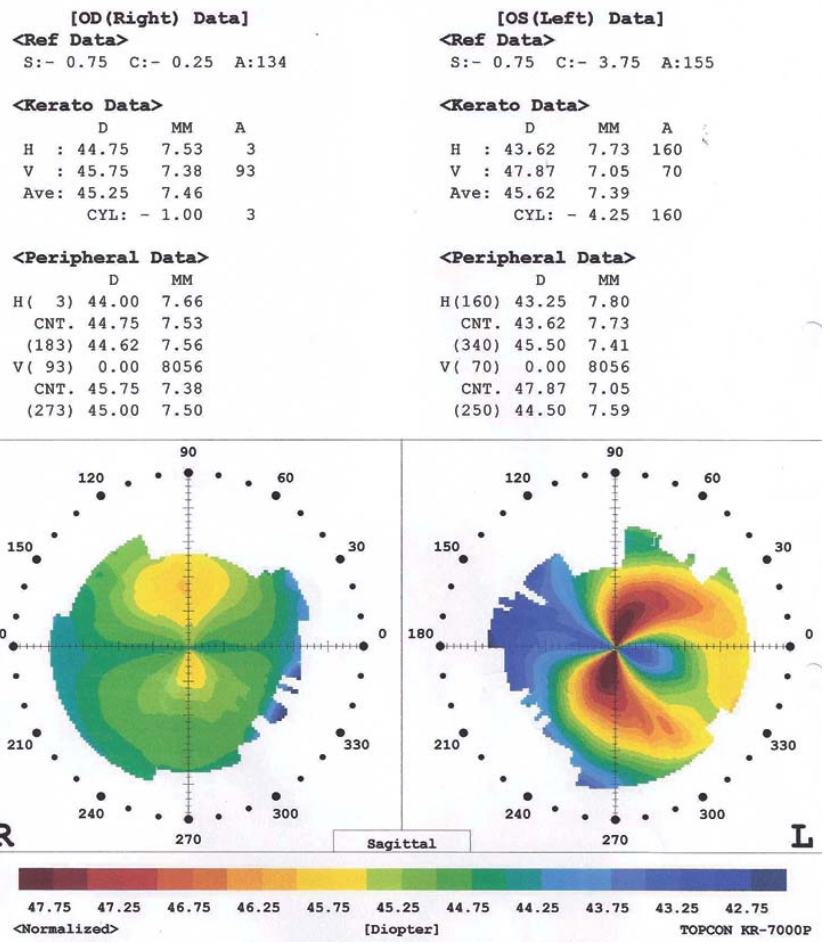

Fig. 9: Postoperative topography, last FU 


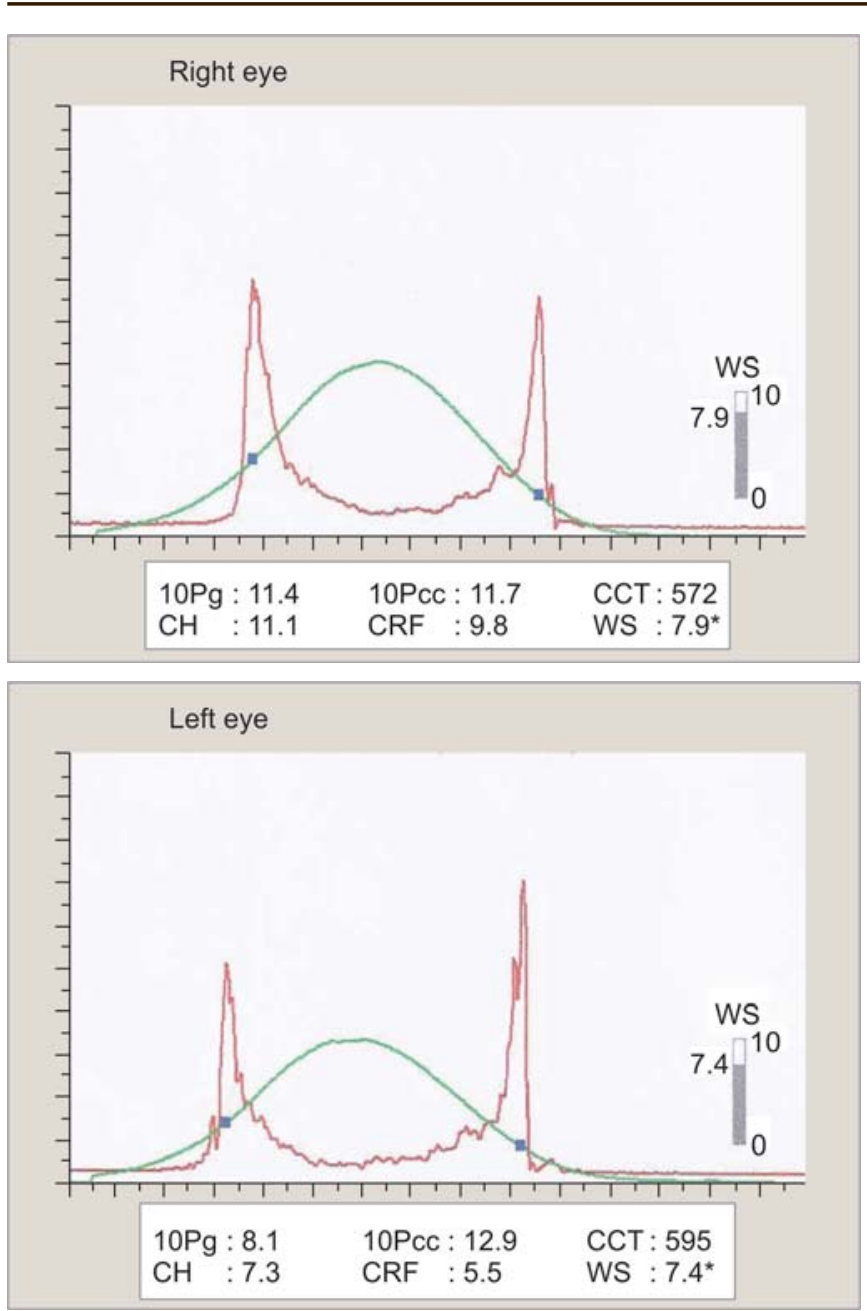

Fig. 10: Ocular response analyzer (ORA), last FU

performed in February 2010. We believe that the method may have an impact on efficacy of the treatment.

A nother factor which may affect the efficacy of the treatment is the cause of the BK. The etiologies reported in the literature for corneal edema comprise more than one pathology (PBK, FED, absolute glaucoma and decompensated corneal graft) although the end result was corneal decompensation. M ost cases suffered from PBK. $C X L$ treatment aims at treating the end result of the pathologic process and not the cause. The surgical techniques were different although most reported cases were treated in the traditional way. D oes the cause have any impact on the surgical outcome? Do causes, such as absolute glaucoma (in which the IOP is continuing to decompensate the endothelium) or PBK (in which the cause of the decompensation is trauma to the endothelium) have different outcomes?

Moreover, reports that show improvement in corneal edema at the last FU are short-term FU and others reports vanishing of the treatment effect during the FU. Hence, one may deduce that the $C X L$ treatment is effective only as a short-term treatment till a PK P or DASEK are performed.
We present a retrospective case of a young male suffering from rapid deterioration of vision as a result of new onset keratoconus followed by a severe diffuse corneal edema (not hydrops), with no tearing of Descemet membrane and no drastic decrease of ECC. Heis an adopted son and we could not detect his family for evaluation of their corneas. His vision was finger counting only, no local or general cause of the edema was found, medical treatment failed and he was candidate for PK P.

Differential diagnosis in our case is:

1. Irridocorneal endothelial syndrome (ICE) but we are missing iris and pupillary changes as well as glaucoma.

2. Endothelitis which is associated with cytomegalovirus, Herpes and EBV . M issing K Ps and iritis. No active viral infection could be detected by blood tests.

3. Posterior polymorphous endothelial dystrophy, however, he is missing Descemet membrane changes.

$C X L$ was offered for two purposes:

1. A rresting the progression of $\mathrm{KC}$.

2. Reducing corneal edema in the hope that his endothelial dysfunction is temporary. In case that the edema recurs inspite of CXL he can undergo DASEK and not PK P because his $K C$ is not a severe and $C X L$ is supposed to arrest it in this nonadvanced stage.

\section{CONCLUSION}

$C X L$ is effective method in reducing corneal edema and its symptoms and improving $V A$ in patients suffering from endothelial decompensation. $\mathrm{No}$ long-term results are reported. In our case the FU is 20 months. Further studies are needed to evaluate the long-term efficacy. In the meantime, this treatment can be offered as a temporary treatment till permanent solution is offered.

\section{REFERENCES}

1. Wollensak G, Spoerl E, Seiler T. Riboflavin/ultraviolet-A induced collagen cross-linking for the treatment of keratoconus A m J Ophthalmol $2003 \mathrm{M}$ ay;135(5):620-27.

2. Spörl E, Raiskup-W olf F, Pillunat LE. Biophysical principles of collagen cross-linking. Klin Monbl A ugenheilkd 2008 Feb;225(2):131-37.

3. G A urich $\mathrm{H}$, Pham DT, Wirbelauer $\mathrm{C}$. Hydration behavior of porcine cornea crosslinked with riboflavin and ultraviolet-A . J Cataract R efract Surg 2007 M ar;33(3):516-21.

4. Ehlers N, Hjortdal J, Nielsen K, Søndergaard A. RiboflavinUVA treatment in the management of edema and nonhealing ulcers of the cornea. J Refract Surg 2009 Sep;25(9):S803-06.

5. Ehlers N , H jortdal J . Riboflavin-ultraviolet light induced crosslinking in endothelial decompensation. A cta O phthal mol 2008 A ug;86(5):549-51.

6. Ghanem RC, Santhiago M R, B erti TB, Thomaz S, N etto M V . Therapeutic effect of corneal cross-linking on symptomatic 
bullous keratopathy. J Cataract R efract Surg 2010 Feb; 36(2): 273-76.

7. Kozobolis V, L abiris G, Gkika M , Sideroudi H, Kaloghianni E, Papadopoulou D, Toufexis G. UV -A collagen cross-linking treatment of bullous keratopathy combined with corneal ulcer. Cornea 2010 Feb;29(2):235-38.

8. W ollensak $\mathrm{G}, \mathrm{A}$ urich $\mathrm{H}$, W irbelauer $\mathrm{C}$, Pham DT. Potential use of riboflavin/UVA cross-linking in bullous keratopathy. Ophthalmic Res 2009;41(2):114-17.

9. K rueger RR, Ramos-Esteban J C, Kanellopoulos A J. 13-Staged intrastromal delivery of riboflavin with UVA cross-linking in advanced bullous keratopathy: L aboratory investigation and first clinical case. Refract Surg 2008 Sep;24(7):S730-36.

10. Cordeiro Barbosa M M , B arbosa J B J r, Hirai FE, H ofling-Lima $A L$. Effect of cross-linking on corneal thickness in patients with corneal edema. Cornea $2010 \mathrm{M}$ ay 6.

11. Gadelha DN, Caval canti BM, B ravo Filho V, Andrade Júnior $N$, B atista N N, Escarião AC. Therapeutic effect of corneal crosslinking on symptomatic bullous keratopalasty. Arq Bras Oftalmol 2009 Jul-Aug;72(4):462-66.

12. Hafezi F, Dejica P, M ajo F. M odified corneal collagen crosslinking reduces corneal oedema and diurnal visual fluctuations in Fuchs dystrophy. B rJ O phthalmol $2010 \mathrm{M}$ ay; 94(5):660-61.

\section{ABOUT THE AUTHORS}

\section{Ramez Barbara (Corresponding Author)}

Department of O phthalmology, B nai Zion M edical Center, 47 Golomb Street, Haifa-31048, Israel, Phone: +972524448687, Fax: +97249815911 e-mail: ramezborbara@gmail.com

\section{Hanna Garzozi}

Department of O phthalmology, B nai Zion M edical Center, Haifa, Israel

\section{Adel Barbara}

Department of Ophthalmology, Hadassah Optimal Medical Center Haifa, Israel

\section{Yossef Pickel}

Department of O phthalmology, Ziv M edical Center, Zefat, I srael

\section{Ankur Barua}

Department of Ophthalmology, Royal Preston Hospital, Lancashire United K ingdom 